\title{
DISPONIBILIDADE DE LUZ EM MACIEIRAS 'FUJI' COBERTAS COM TELAS ANTIGRANIZO E SEUS EFEITOS SOBRE A FOTOSSÍNTESE, O RENDIMENTO E A QUALIDADE DOS FRUTOS ${ }^{1}$
}

\author{
CASSANDRO VIDAL TALAMINI DO AMARANTE², CRISTIANO ANDRÉ STEFFENS ${ }^{3}$, \\ AQUIDAUANA MIQUELOTO ${ }^{4}$, ODIMAR ZANUZO ZANARDI ${ }^{5}$, \\ HENRIQUE PESSOA DOS SANTOS
}

\begin{abstract}
RESUMO - Em pomares de macieira, o dano de granizo pode ser evitado através da cobertura das plantas com telas. Todavia, as telas alteram a intensidade e a qualidade da luz solar e, assim, podem comprometer o rendimento e a qualidade dos frutos. Este trabalho objetivou avaliar estes aspectos em macieiras 'Fuji', cobertas com telas nas cores branca e preta. A tela preta ocasionou maiores reduções na densidade de fluxo de fótons fotossinteticamente ativos (DFFFA) disponíveis às plantas $(24,8 \%)$ em relação à tela branca $(21,2 \%)$. O interior do dossel das plantas sob tela, especialmente quando cobertas com tela preta, recebeu radiação com menores valores da relação vermelho:vermelho distante $(\mathrm{V}: \mathrm{Vd})$ em relação às plantas descobertas. Somente sob tela preta, a magnitude das reduções na DFFFA e na relação V:Vd da luz foi capaz de aumentar a área média e a área específica das folhas e reduzir a taxa fotossintética potencial, reduzindo assim o rendimento (número e peso de frutos por $\mathrm{cm}^{2}$ de seção transversal de tronco) e a coloração vermelha dos frutos. As telas antigranizo branca e preta reduziram a incidência de queimadura de sol, porém não afetaram a severidade de "russeting" e o número de sementes por fruto.
\end{abstract}

Termos para indexação: intensidade e qualidade de luz, cor do fruto, queimadura de sol, "russeting", Malus domestica.

\section{LIGHT SUPPLY TO 'FUJI' APPLE TREES COVERED WITH HAIL PROTECTION NETS AND ITS EFFECTS ON PHOTOSYNTHESYS, YIELD AND FRUIT QUALITY}

\begin{abstract}
In apple orchards, hail damage can be avoided by covering the plants with nets. However, the nets might change light intensity and quality supplied to the plants and, therefore, affect yield and quality of the fruit. This research was carried out to assess these aspects on 'Fuji' apples trees uncovered and covered with white and black nets. The black net caused more substantial reduction of photosynthetic photons flux density (PPFD) available to the plants during the day (24.8\%) than the white one (21.2\%). The inner part of plants canopy covered by nets, especially under black net, received light with a lower red:far red ratio (R:FR), in comparison to uncovered plants. Only under black net, the reductions of PPFD and R:FR of the light supplied to the plants increased the mean area and the specific area of the leaves and reduced the potential photosynthesis, leading to a reduction of yield (number and weight of fruits per $\mathrm{cm}^{-2}$ of trunk cross section area) and the red color of the fruit. The white and black hail protection nets reduced the incidence of sunburn but had no effect on russeting severity and number of seeds/fruit.
\end{abstract}

Index terms: light intensity and quality, fruit skin color, sunburn, russeting, Malus domestica.

\footnotetext{
'(Trabalho 134-08). Recebido em: 02-06-2008. Aceito para publicação em:14-05-2009.

${ }^{2}$ Ph.D., bolsista de produtividade em pesquisa do CNPq. Professor do Departamento de Agronomia, Centro de Ciências Agroveterinárias (CAV), Universidade do Estado de Santa Catarina (UDESC). Cx. Postal 281, CEP 88520-000, Lages-SC. Autor para correspondência. E-mail: amarante@cav.udesc.br

${ }^{3}$ Dr., Professor do Departamento de Agronomia, CAV/UDESC, Lages, SC. E-mail: steffens@cav.udesc.br

${ }^{4}$ Acadêmicos do Curso de Agronomia, Bolsistas de Iniciação Científica do CNPq. CAV/UDESC, Lages-SC. E-mail: quimique@bol. com.br; odimarzanardi@yahoo.com.br

${ }^{5}$ Dr., Embrapa Uva e Vinho, Rua Livramento, 515, Caixa Postal 130, CEP 95700-000 Bento Gonçalves-RS. E-mail: henrique@cnpuv. embrapa.br
} 


\section{INTRODUÇÃO}

O cultivo da macieira (Malus domestica Borkh) ocorre em regiões de altitude que propiciam condições climáticas adequadas para a produção e a qualidade dos frutos. Essas mesmas condições climáticas de altitude favorecem a ocorrência de granizo, com prejuízos significativos aos produtores (Yuri, 2003).

O granizo é formado na parte superior de nuvens do tipo cúmulo-nimbos, onde a temperatura é menor e favorece a transformação de gotículas de água em partículas de gelo, com diâmetro médio de 1,5 a $2 \mathrm{~cm}$, que pode variar de 0,5 a $20 \mathrm{~cm}$ (Martinez et al., 2001). O granizo ocorre de forma localizada, não atinge grandes áreas, mas pode ocasionar significativa redução na qualidade e quantidade de frutos destinados à comercialização, assim como danos às árvores em formação e em produção, o que compromete as produções futuras (Leite et al., 2002). Isto tem levado à adoção, por parte dos produtores, de alternativas para a administração dos prejuízos, que incluem a utilização de foguetes antigranizo, geradores de solo, o seguro agrícola e a cobertura dos pomares com telas antigranizo (Yuri, 2003).

A cobertura das linhas de plantas com telas é considerada o método mais seguro de proteção contra o granizo (Poldervaart, 2006; Leite et al., 2002) e apesar de seu elevado custo, estimado entre $\mathrm{R} \$ 15$ mil e R\$ 20 mil por hectare, seu emprego tem aumentado em pomares de macieiras nos Estados de Santa Catarina e Rio Grande do Sul (Amarante et al., 2007). A tela antigranizo é confeccionada com tramas finas, que proporcionam maior resistência mecânica, capaz de suportar fortes chuvas de granizo, recebe tratamento de proteção contra radiação ultravioleta (UV) e sua durabilidade alcança cerca de 15 anos para a tela preta e 10 anos para a tela branca (Tassara \& Battaglia, 1992). As empresas que comercializam telas antigranizo para utilização em pomares de maçãs informam que os níveis de sombreamento ocasionados são de $8-12 \%$ para telas brancas e de $18-25 \%$ para telas pretas.

A tela antigranizo reduz a radiação solar incidente sobre as plantas, e pode por isso interferir na fotossíntese, produção e qualidade dos frutos. A intensidade desses efeitos está relacionada com o grau de sombreamento (dependente da coloração e da malha da tela), da combinação cultivar/porta-enxerto, da densidade de plantio, do sistema de manejo e condução das plantas e da região de produção (Gardner \& Fletcher, 1990; Tassara \& Battaglia, 1992; Chen et al., 1997; Widmer, 2001; Leite et al., 2002; Middleton \& McWaters, 2002; Stampar et al., 2002; Warnier,
2004; Amarante et al., 2007).

Este trabalho foi conduzido em pomar de macieira 'Fuji', com a finalidade de estudar os efeitos do emprego de telas antigranizo, nas cores branca e preta, sobre a intensidade e qualidade da luz disponibilizada às plantas, e os seus impactos sobre a fotossíntese, rendimento e qualidade dos frutos.

\section{MATERIAL E MÉTODOS}

O experimento foi conduzido em pomar comercial no município de Vacaria-RS (situado a $930 \mathrm{~m}$ de altitude, latitude $28^{\circ} 22^{\prime} 52,1^{\prime \prime} \mathrm{S}$ e longitude $\left.50^{\circ} 50^{\prime} 46,3^{\prime \prime} \mathrm{W}\right)$, durante três ciclos produtivos (2003/04 a 2005/06). Foram utilizadas macieiras 'Fuji', com 8-10 anos de idade, sobre porta-enxerto M9, conduzidas com líder central, em sistema de alta densidade, com espaçamento de 3,5 m x 0,8 m.

O delineamento experimental foi em blocos ao acaso, com cinco repetições, sendo cada planta uma repetição. Os tratamentos consistiram no controle (plantas não cobertas com tela antigranizo) e na cobertura das plantas com telas antigranizo (Sombrite ${ }^{\circledR}$, Empresa Equipesca) nas cores branca e preta, ambas com malha de 4,0 x 7,0 $\mathrm{mm}$. As plantas dos tratamentos com tela permaneceram cobertas durante todo o ciclo (dormência e crescimento vegetativo), não sendo retiradas durante a floração. As telas branca e preta foram montadas ao longo da linha de plantio (em outubro de 1999), seguindo a orientação norte-sul, no formato de cobertura de duas águas, ficando uma abertura entre filas de 20-30 $\mathrm{cm}$, visando a permitir a queda de pedras de granizo entre as filas de plantas. Foram cobertas nove linhas de plantas, ao longo de $140 \mathrm{~m}$ de extensão, com cada uma das telas utilizadas (branca e preta). Foram utilizadas cinco plantas da linha central, na porção mediana da extensão total coberta com as telas, para as avaliações de fotossíntese, rendimento e qualidade de frutos, além de cinco plantas na área descoberta, distante cinco linhas da área coberta.

A densidade de fluxo de fótons fotossinteticamente ativos (DFFFA; $\lambda=400-700 \mathrm{~nm}$ ) na região entre as linhas de plantas, nas áreas cobertas ou não com telas antigranizo, foi determinada com um sensor quântico plano marca Li-Cor, modelo LI-190, sensível às faixas de comprimento de onda entre 400 e $700 \mathrm{~nm}$, conectado a um datalogger modelo LI-1400, no período de $05 \mathrm{~h}$ às $20 \mathrm{~h}$. A irradiância espectral (IE; $\mathrm{W} \mathrm{m}^{-2} \mathrm{~nm}^{-1}$ ), na faixa do vermelho distante (Vd), foi caracterizada próximo do comprimento de onda de $735 \mathrm{~nm}$, e o vermelho (V), ao redor de $645 \mathrm{~nm}$. Foram determinadas com um espectrorradiômetro portátil marca Li-Cor, modelo LI-1800, na altura do 
terço médio do dossel das plantas, na entrelinha e no interior do dossel das plantas, próximo ao meio-dia. A IE nesses comprimentos de onda foi utilizada para o cálculo da relação vermelho:vermelho distante (V:Vd) da radiação (Awad et al., 2001).

A curva de taxa de assimilação líquida de $\mathrm{CO}_{2}\left(A, \mu \mathrm{mol} \mathrm{CO}{ }_{2} \mathrm{~m}^{-2} \mathrm{~s}^{-1}\right)$ em resposta à densidade de fluxo de fótons fotossinteticamente ativos (DFFFA), em valores de $0 ; 90 ; 250 ; 600$ e $1.000 \mu \mathrm{mol}$ de fótons $\mathrm{m}^{-2} \mathrm{~s}^{-1}$, foi determinada com o analisador de gás por infravermelho (IRGA) portátil, marca LiCor, modelo LI-6400, operando em sistema aberto, equipado com fonte de luz modelo LI-6400-02B. As avaliações foram efetuadas em uma folha por planta, próximo ao meio-dia, utilizando sempre a folha do terço médio das plantas, não sombreada por outras e oposta aos frutos. A partir da curva de resposta de $A$ em função da DFFFA, ajustada através da função hiperbólica $A=a+\left[\left(A_{\text {máx }} \times\right.\right.$ DFFFA $) /(b+$ DFFFA $\left.)\right]$, em que $A_{\text {máx }}$ é a taxa máxima de fotossíntese e $a$ e $b$ são coeficientes de ajuste da equação, foi possível calcular a respiração no escuro $\left(\mathrm{R}_{\mathrm{e}}\right.$, igual ao coeficiente $a$ da equação), o ponto de compensação de luz ( $\Gamma$, correspondente ao valor de DFFFA, quando $A$ é igual a zero) e estimar a eficiência quântica aparente $\left(\Phi_{\mathrm{a}} ; \mu \mathrm{mol} \mathrm{CO} / \mu \mathrm{mol}\right.$ fótons), conforme descrito por Amarante et al. (2007).

As avaliações de DFFFA, IE e $A$ foram feitas em uma condição de céu completamente limpo, no dia 19-01-2006.

Em 24 de janeiro de 2006 (cerca de um mês antes da colheita comercial dos frutos), foram feitas amostragens de 20 folhas por repetição, no terço médio de ramos do ano, sendo as mesmas avaliadas em termos de teor total de clorofila $\left(\mathrm{mg} \mathrm{m}^{-2}\right)$, área foliar (com um integrador de área foliar LI-COR modelo LI-3050A) e massa seca (após secagem durante 72 $\mathrm{h}$ a $65^{\circ} \mathrm{C}$ e pesagem em balança analítica). A área foliar específica $\left(\mathrm{cm}^{2} \mathrm{~g}^{-1}\right)$ foi calculada pela divisão da área $\left(\mathrm{cm}^{2}\right)$ pela massa seca ( $\mathrm{g}$ ) foliar. A extração e a quantificação do teor total de clorofila foram feitas segundo metodologia descrita por Passos (1996).

Foram colhidos todos os frutos de cada planta na maturação comercial, nas três safras de execução do trabalho, sendo os mesmos contados e pesados, para a determinação do rendimento (número e peso de frutos $/ \mathrm{cm}^{2}$ de seção transversal de tronco) e do peso médio de frutos $(\mathrm{g})$. Foram selecionados 30 frutos de cada planta, com tamanho uniforme (calibre 120-135), os quais foram avaliados quanto à coloração, percentagem de frutos com queimadura de sol, severidade de "russeting" $\left(\mathrm{cm}^{2}\right.$ fruto $\left.^{-1}\right)$ e número de sementes/fruto. A coloração foi avaliada em termos de superfície colorida do fruto (percentagem de cor vermelha), através de análise subjetiva visual, e de valor de ângulo 'hue' $\left(h^{o}\right)$, determinado com o auxílio de um colorímetro Minolta modelo CR 400, nos lados mais e menos expostos à luz (correspondentes aos lados com maior e menor intensidade de coloração vermelha, respectivamente).

Os dados foram submetidos às análises de regressão não linear e de variância, e as médias de tratamentos, comparadas pelo teste de Tukey $(\mathrm{p}<0,05)$, com o programa SAS

\section{RESULTADOS E DISCUSSÃO}

Houve redução substancial na DFFFA acumulada ao longo do dia, nas áreas cobertas com telas, comparativamente à área descoberta (Tabela 1). Porém, a tela preta ocasionou maior redução na DFFFA $(24,8 \%)$ em relação à área descoberta do que a tela branca $(21,2 \%)$ na entrelinha. Widmer (2001) e Middleton \& McWaters (2002) também observaram maiores reduções na DFFFA em pomares de macieiras sob telas antigranizo pretas do que sob telas brancas.

O sombreamento ocasionado pelas telas antigranizo resultou em menores valores de respiração no escuro $\left(\mathrm{R}_{\mathrm{e}}\right)$, ponto de compensação de luz $(\Gamma)$ e taxa máxima de fotossíntese $\left(A_{\text {máx }}\right)$ nas folhas, em resposta ao aumento na DFFFA (Figura 1). $\mathrm{AR}_{\mathrm{e}}$ (coeficiente $a$ da equação hiperbólica ajustada) foi de 1,$17 ; 0,87$ e $0,67 \mu \mathrm{mol}$ de $\mathrm{CO}_{2} \mathrm{~m}^{-2} \mathrm{~s}^{-1}$ nos tratamentos-controle, tela branca e tela preta, respectivamente. Os valores de $\Gamma$ foram de 18,5 ; 11,7 e $9,1 \mu \mathrm{mol}$ de fótons $\mathrm{m}^{-2} \mathrm{~s}^{-1}$ nos tratamentoscontrole, tela branca e tela preta, respectivamente. Plantas descobertas e plantas cobertas com telas branca e preta apresentaram valores de $A_{\text {máx }}$ correspondentes a 23,$6 ; 15,0$ e $15,5 \mu \mathrm{mol}$ de $\mathrm{CO}_{2} \mathrm{~m}^{-2} \mathrm{~s}^{-1}$, respectivamente. A eficiência quântica aparente $\left(\Phi_{\mathrm{a}}\right)$ foi ligeiramente inferior em plantas descobertas $\left(0,060 \mu \mathrm{mol} \mathrm{CO}_{2} / \mu \mathrm{mol}\right.$ fótons $)$, comparativamente às plantas cobertas com telas branca e preta $\left(0,065\right.$ e $0,064 \mu \mathrm{mol} \mathrm{CO}_{2} / \mu \mathrm{mol}$ fótons, respectivamente). Os valores do coeficiente $b$ das equações hiperbólicas ajustadas (DFFFA correspondente à metade da $A_{\text {máx }}$ ) foram maiores nas plantas do tratamento-controle $(354,96 \mu \mathrm{mol}$ de fótons $\mathrm{m}^{-2} \mathrm{~s}^{-1}$ ) do que em plantas cobertas com telas branca $\left(190,35 \mu \mathrm{mol}\right.$ de fótons $\left.\mathrm{m}^{-2} \mathrm{~s}^{-1}\right)$ e preta $\left(202,07 \mu \mathrm{mol}\right.$ de fótons $\left.\mathrm{m}^{-2} \mathrm{~s}^{-1}\right)$ (Figura 1).

Os dados mostram que o sombreamento ocasionado pelas telas aumentou a eficiência fotossintética em baixos valores de DFFFA, caracterizado pelos menores valores de $\mathrm{R}_{\mathrm{e}}, \Gamma$ e coeficiente $b$ das equações hiperbólicas ajustadas, mas ocasionou 
menor $A_{\text {máx }}$. A pequena redução da $\mathrm{R}_{\mathrm{e}} \mathrm{em}$ folhas sombreadas é uma estratégia importante para a redução do $\Gamma$, por aumentar a eficiência de captação e uso da pouca luz disponível em ambiente sombreado (Dussi et al., 2005). No entanto, ocorre redução nos valores de $A_{\text {máx }}$ em plantas sombreadas pela tela, como resultado da maior limitação imposta pelas reações de carboxilação ao incremento na fotossíntese, em elevados valores de DFFFA, comparativamente às plantas descobertas (Lambers et al., 1998).

A utilização de telas não afetou o teor foliar de clorofila total (Tabela 2). No entanto, plantas cobertas com tela antigranizo preta apresentaram maiores valores de área foliar média e área foliar específica em relação às plantas descobertas (Tabela 2). O aumento no teor de clorofila e nos valores de área foliar média e área foliar específica reflete adaptações das plantas ao sombreamento, visando a aumentar a eficiência fotossintética em condições da baixa DFFFA (Lambers et al., 1998; Dussi et al., 2005). Em macieiras 'Royal Gala' cobertas com tela antigranizo preta, além do aumento na área foliar específica em relação às plantas descobertas, ocorreu ainda aumento no teor foliar de clorofila total (Amarante et al., 2007). Isso mostra que o aumento no teor de clorofila em resposta ao sombreamento depende da cultivar, enquanto a expansão na área foliar parece representar um mecanismo comum de macieiras visando a aumentar a eficiência da interceptação da radiação nas condições de sombreamento impostas pelas telas antigranizo. Todavia, como plantas cobertas com tela antigranizo, especialmente sob tela preta, apresentaram folhas com maior área e menor espessura (com maior área foliar específica), isso resulta em melhor aproveitamento da radiação incidente ao longo de todo o mesófilo foliar, aumentando assim a eficiência fotossintética em condições de baixa disponibilidade de luz. Isso é constatado pela redução do $\Gamma$ e do coeficiente $b$ das equações hiperbólicas ajustadas, relacionando $A$ ao incremento na DFFFA em plantas cobertas com telas antigranizo (Figura 1), especialmente na cor preta, que ocasiona maior sombreamento (Tabela 1).

A maior restrição de radiação fotossinteticamente ativa imposta pela tela preta, mesmo com as alterações verificadas na fotossíntese e no desenvolvimento foliar para adaptação a menor disponibilidade de luz, resultou em redução no número e peso de frutos por $\mathrm{cm}^{2}$ de seção transversal de tronco, em relação às plantas descobertas (Tabela 2). No entanto, em plantas cobertas com tela branca, o rendimento não diferiu das plantas descobertas (Tabela 2). O peso médio de frutos não diferiu do controle em plantas cobertas com telas branca e preta (Tabela 2).

Os menores valores de DFFFA e da relação $\mathrm{V}: \mathrm{Vd}$ no interior do dossel sob tela antigranizo preta (Tabela 1) resultaram em frutos com menor percentagem de coloração vermelha e menor $h^{o}$ nos lados sombreado e exposto à luz dos frutos (Tabela 3 ). $\mathrm{O}$ acúmulo de antocianinas ocasiona redução no $h^{o}$, e reflete a mudança de cor verde para vermelha, sendo este processo menos intenso em frutos de plantas cobertas com tela preta. Frutos de plantas cobertas com tela branca não diferiram das plantas descobertas quanto à coloração.

A redução na cor vermelha dos frutos sob tela preta confirma resultados obtidos por outros autores, sobre os efeitos da quantidade e qualidade da radiação na coloração de maçãs (Gardner \& Fletcher, 1990; Awad et al., 2001; Leite et al., 2002; Stampar et al., 2002; Amarante et al., 2007). Segundo Awad et al. (2001), valores da relação V:Vd inferiores a 1,0 , observados no interior do dossel de macieiras 'Jonagold', reduziram o conteúdo de cianidina 3-galactosídeo (antocianina), quercetina 3-glicosídeo e flavonoides totais, resultando em deficiente coloração vermelha nos frutos.

Os tratamentos não afetaram significativamente a severidade de "russeting" (área de sintoma/ fruto), que foi de 2,6; 2,8 e 3,3 $\mathrm{cm}^{2}$ fruto $^{-1}$ em plantas descobertas e em plantas cobertas com telas antigranizo branca e preta, respectivamente (valores médios de três safras). Amarante et al. (2007) também reportaram ausência de efeito da cobertura de macieiras 'Royal Gala' com telas antigranizo na severidade de "russeting". O emprego de telas pode aumentar a severidade de "russeting", devido à restrição no movimento de ar neste ambiente, e permitir, assim, maior umidade relativa (Middleton \& McWaters, 2002), que está associada à manifestação do distúrbio em maçãs. No entanto, Leite et al. (2002) e Stampar et al. (2002) observaram menor severidade de "russeting" em plantas cobertas pela tela em relação a plantas descobertas, como resultado da diminuição da radiação solar, que é um dos fatores que reduzem a manifestação do distúrbio.

Houve menor incidência de queimadura de sol $(\mathrm{p}<0,05)$ em frutos de plantas cobertas com telas antigranizo branca $(9,2 \%)$ e preta $(6,9 \%)$ em comparação às plantas descobertas (13,7\%) (valores médios de três safras). Porém, os frutos sob tela preta apresentaram menor incidência de queimadura de sol, em relação aos frutos sob tela branca, em virtude de a tela preta promover maior redução de luminosidade (Tabela 1). Isto confirma dados de outros autores (Tassara \& Battaglia, 1992; Leite et al., 2002; Middleton \& McWaters, 2002; Stampar et al., 2002; Amarante et 
al., 2007) e mostra que a redução na intensidade luminosa em plantas sob tela reduz a incidência de frutos com queimadura de sol na cultura da macieira.

A tela antigranizo pode comprometer o processo de polinização entomófila das macieiras (Leite et al., 2002; Yuri, 2003) e, portanto, a frutificação efetiva e o rendimento de frutos. Entretanto, o número de sementes por fruto não diferiu entre plantas descobertas e plantas cobertas com telas (com valores médios em todos os tratamentos de 6,6 sementes por fruto), evidência de que a tela não interferiu com a atividade polinizadora de abelhas. Resultados semelhantes foram reportados por Amarante et al. (2007) em macieiras 'Royal Gala' cobertas com telas antigranizo. Portanto, a redução no rendimento de frutos, expressa em $\mathrm{g}$ por $\mathrm{cm}^{2}$ de seção transversal de tronco, em plantas cobertas com tela preta (Tabela 2), pode ser consequência da redução no suprimento de luz, que reduz o acúmulo de reservas e pode comprometer a diferenciação de gemas reprodutivas e a frutificação efetiva (Chen et al., 1997; Middleton \&
McWaters, 2002).

Os resultados obtidos mostram que a tela branca é mais indicada na proteção ao granizo em macieiras 'Fuji', apesar de sua menor durabilidade, já que permite maior intensidade e melhor qualidade de luz, necessários para a fotossíntese e o acúmulo de antocianinas na casca dos frutos, em comparação com a tela preta. Todavia, mesmo com emprego de tela branca, cuidados especiais devem ser tomados quanto ao manejo e condução, especialmente no que diz respeito a podas, visando a permitir maior penetração de luz no dossel das plantas e assim aumentar a cor vermelha dos frutos, especialmente em regiões e safras agrícolas com condições climáticas desfavoráveis ao acúmulo de antocianinas nos frutos (Awad et al., 2001). Aliado a isto, o plantio de mutações de 'Fuji' com maior coloração vermelha, como é o caso de 'Fuji Kiku' e 'Fuji Suprema', sobre porta-enxerto anão, pode tornar o emprego de tela antigranizo branca uma alternativa viável e de baixo impacto no comprometimento da qualidade dos frutos.

TABELA 1 - Densidade de fluxo de fótons fotossinteticamente ativos (DFFFA) acumulados ao longo do dia e valor da relação vermelho:vermelho distante (V:Vd) da radiação quantificada nas regiões da entrelinha e do interior do dossel, em macieiras 'Fuji' descobertas (controle) ou cobertas com telas antigranizo branca e preta.

\begin{tabular}{cccc}
\hline Tratamento & Região do pomar & $\begin{array}{c}\text { DFFFA acumulada } \\
\text { (molde fótons } \mathrm{m}^{-2} \text { ) }\end{array}$ & $\begin{array}{c}\text { Relação } \\
\mathrm{V}: \mathrm{Vd}\end{array}$ \\
\hline Controle & Entrelinha & 61,8 & $1,51 \mathrm{a}$ \\
Tela Branca & Interior do dossel & -- & $0,98 \mathrm{~A}$ \\
& Entrelinha & 48,7 & $1,38 \mathrm{~b}$ \\
Tela Preta & Interior do dossel & -- & $0,89 \mathrm{AB}$ \\
& Entrelinha & 46,5 & $1,43 \mathrm{~b}$ \\
& Interior do dossel & -- & $0,79 \mathrm{~B}$ \\
\hline
\end{tabular}

Valores $(n=5)$ da relação $V: V d$ seguidos da mesma letra nas colunas, minúsculas para a região da entrelinha e maiúsculas para a região do interior do dossel, não diferem entre si, pelo teste de Tukey $(\mathrm{p}<0,05)$.

TABELA 2 - Conteúdo de clorofila, área média e área específica das folhas, e rendimento e peso médio de frutos, em macieiras 'Fuji' descobertas (controle) ou cobertas com telas antigranizo branca e preta.

\begin{tabular}{|c|c|c|c|c|c|c|}
\hline \multirow{2}{*}{ Tratamento } & \multirow{2}{*}{$\begin{array}{c}\text { Clorofila } \\
\text { foliar } \\
\left(\mathrm{g} \mathrm{m}^{-2}\right)\end{array}$} & \multirow{2}{*}{$\begin{array}{l}\text { Área foliar } \\
\text { média } \\
\left(\mathrm{cm}^{2}\right)\end{array}$} & \multirow{2}{*}{$\begin{array}{c}\text { Área foliar } \\
\text { específica } \\
\left(\mathrm{cm}^{2} \mathrm{~g}^{-1}\right)\end{array}$} & \multicolumn{2}{|c|}{ Rendimento } & \multirow{2}{*}{$\begin{array}{l}\text { Peso médio de } \\
\text { frutos }(\mathrm{g})\end{array}$} \\
\hline & & & & $\begin{array}{c}\left(\mathrm{n}^{\circ} \mathrm{de} \text { frutos } / \mathrm{cm}^{2} \mathrm{de}\right. \\
\text { seção transversal de } \\
\text { tronco) }\end{array}$ & $\begin{array}{l}\text { (g de frutos } / \mathrm{cm}^{2} \mathrm{de} \\
\text { seção transversal de } \\
\text { tronco) }\end{array}$ & \\
\hline Controle & $208,28 \mathrm{a}$ & $35,40 \mathrm{~b}$ & $119,43 \mathrm{~b}$ & $3,88 \mathrm{a}$ & $509,90 \mathrm{a}$ & $139,56 \mathrm{ab}$ \\
\hline Tela Branca & $218,98 \mathrm{a}$ & $36,73 \mathrm{ab}$ & $138,72 \mathrm{ab}$ & $2,98 \mathrm{ab}$ & $379,77 \mathrm{ab}$ & $135,35 \mathrm{~b}$ \\
\hline Tela Preta & $217,69 a$ & $41,44 \mathrm{a}$ & $142,91 \mathrm{a}$ & $2,36 \mathrm{~b}$ & $321,80 \mathrm{~b}$ & $147,22 \mathrm{a}$ \\
\hline C.V. $(\%)$ & 8,55 & 9,94 & 10,75 & 31,15 & 28,72 & 4,96 \\
\hline
\end{tabular}

Valores seguidos da mesma letra, nas colunas, não diferem entre si, pelo teste de Tukey $(\mathrm{p}<0,05)$. 
TABELA 3 - Cor vermelha e ângulo 'hue' $\left(h^{\circ}\right)$ dos frutos em macieiras 'Fuji' descobertas (controle) ou cobertas com telas antigranizo branca e preta.

\begin{tabular}{|c|c|c|c|}
\hline \multirow{2}{*}{ Tratamento } & \multirow{2}{*}{$\begin{array}{c}\text { Cor vermelha } \\
(\%)\end{array}$} & \multicolumn{2}{|c|}{$h^{o}$} \\
\hline & & Lado sombreado & Lado exposto à luz \\
\hline Controle & $58,57 \mathrm{a}$ & $101,44 \mathrm{~b}$ & $50,87 \mathrm{~b}$ \\
\hline Tela Branca & $55,50 \mathrm{a}$ & $101,02 \mathrm{~b}$ & $48,00 \mathrm{~b}$ \\
\hline Tela Preta & $46,88 \mathrm{~b}$ & $105,64 \mathrm{a}$ & $60,19 a$ \\
\hline C.V. (\%) & 12,27 & 6,70 & 31,16 \\
\hline
\end{tabular}

Valores seguidos da mesma letra, nas colunas, não diferem entre si, pelo teste de Tukey $(\mathrm{p}<0,05)$.

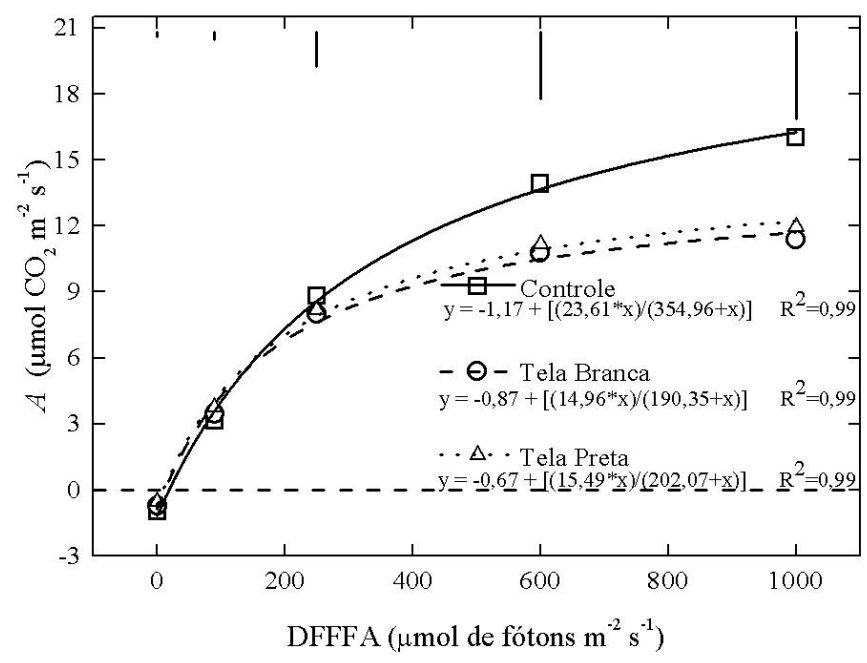

FIGURA 1 - Taxa foliar de assimilação líquida de $\mathrm{CO}_{2}$ (A) em função da densidade de fluxo de fótons fotossinteticamente ativos (DFFFA) em macieiras 'Fuji' descobertas (controle) ou cobertas com telas antigranizo nas cores branca e preta. As diferenças mínimas significativas entre tratamentos, para cada nível de DFFFA, indicadas no interior da figura (barras verticais), foram calculadas pelo teste de Tukey $(\mathrm{p}<0,05)$.

\section{CONCLUSÃO}

O emprego de tela antigranizo preta ocasiona maior redução na intensidade e na qualidade da luz disponível às plantas do que a tela branca. Em consequência disso, a tela antigranizo preta provoca redução na coloração vermelha dos frutos, bem como um decréscimo na taxa fotossintética potencial, levando assim a uma redução no rendimento de frutos. As telas antigranizo branca e preta reduzem a incidência de queimadura de sol, porém não afetam a severidade de "russeting" e o número de sementes por fruto. A tela antigranizo branca é mais indicada na proteção ao granizo em macieiras 'Fuji', já que permite maior intensidade e melhor qualidade de luz, necessários para a fotossíntese e o acúmulo de antocianinas na casca dos frutos, em comparação com a tela preta.

\section{AGRADECIMENTOS}

Os autores agradecem ao Conselho Nacional de Desenvolvimento Científico e Tecnológico (CNPq), pelo apoio financeiro a este projeto.

\section{REFERÊNCIAS}

AMARANTE, C.V.T.; STEFFENS, C.A.; MOTA, C.S.; SANTOS, H.P. Radiação, fotossíntese, rendimento e qualidade de frutos em macieiras 'Royal Gala' cobertas com telas antigranizo. Pesquisa Agropecuária Brasileira, Brasília, v.42, n.7, p.925-931, 2007. 
AWAD, M.A.; WAGENMAKERS, P.S.; JAGER, A. de. Effects of light on flavonoid and chlorogenic acid levels in the skin of 'Jonagold' apples. Scientia Horticulturae, Amsterdam, v.88, p.289-298, 2001.

CHEN, K.; HU, G.Q.; LENZ, F. Training and shading effects on vegetative and reproductive growth and fruit quality of apple. Gartenbauwissenschaft, Munique, v. 62, p. 207-213, 1997.

DUSSI, M.C.; GIARDINA, G.; SOSA, D.; GONZALEZ-JUNYENT, R.; ZECCA, A.; REEB, $P$. Shade nets effect on canopy light distribution and quality of fruit and spur leaf on apple cv. Fuji. Spanish Journal of Agricultural Research, Madri, v.3, p.253-260, 2005.

GARDNER, R.A.W.; FLETCHER, C.A. Hail protection systems for deciduous fruit trees. Deciduous Fruit Grower, Cape Town, v.40, p.206212, 1990.

LAMBERS, H.; CHAPIN, F.S.; PONS, T.L. Plant physiological ecology. New York: Springer-Verlag, 1998. 540p.

LEITE, G.B.; PETRI, J.L.; MONDARDO, M. Efeito da tela antigranizo em algumas características dos frutos de macieira. Revista Brasileira de Fruticultura, Jaboticabal, v. 24, p. 714-716, 2002.

MARTINEZ, J.F.; MILLAN, M.; GARCIA, N.; LOPEZ, F.V.; DELGADO, A.; GARCIA, R.; MIDDLETON, S.; McWATERS, A. Hail netting of apple orchards: Australian experience. Compact Fruit Tree, Middleburg, v.35, p.51-55, 2002.
PASSOS, L.P. Métodos analíticos e laboratoriais em fisiologia vegetal. Coronel Pacheco: Embrapa Gado de Leite, 1996. 223p.

POLDERVAART, G. Hail nets: reliable method of hail protection. Fruitteelt, Den Haag, v.96, p. 1213, 2006.

STAMPAR, F.; VEBERIC, R.; ZADRAVEC, P.; HUDINA, M.; USENIK, V.; SOLAR, A.; OSTERC, G. Yield and fruit quality of apples cv. 'Jonagold' under hail protection nets. Gartenbauwissenschaft, Stuttgart, v.67, p.205-210, 2002.

TASSARA, M.A.; BATTAGLIA, M. Ensayo de una red plastica antigranizo en manzanos en el Alto Valle de Rio Negro. Rivista di Agricoltura Subtropicale e Tropicale, Firenze, v.86, p.375-384, 1992.

WARNIER, O. The influence of an anti-hail net on the quality of fruits and their production. Fruit Belge, Liège, v.72, p.86-93, 2004.

WIDMER, A. Light intensity and fruit quality under hail protection nets. Acta Horticulturae, Leuven, n. 557, p. 421-426, 2001.

YURI, H. M. Gestão de risco de granizo pelo seguro e outras alternativas: estudo de caso em pomares de maçã de Santa Catarina. 2003. 145 f. Dissertação (Mestrado)- Escola Superior de Agricultura Luiz de Queiroz, Universidade de São Paulo, Piracicaba, 2003. 